\title{
Relationships between retinal arteriole anatomy and aortic geometry and function and peripheral resistance in hypertensives
}

\author{
David Rosenbaum ${ }^{1,2,3}$, Nadjia Kachenoura ${ }^{2}$, Edouard Koch ${ }^{4}$, Michel Paques ${ }^{4}$, Philippe Cluzel ${ }^{2,3,5}$, \\ Alban Redheuil ${ }^{2,3,5}$ and Xavier Girerd ${ }^{1}$
}

\begin{abstract}
Microvascular remodeling and large artery stiffness are key determinants of cardiovascular hemodynamics and can now be studied with new non-invasive methods. Our objective was to study the relationships between retinal arteriole anatomy and aortic geometry and function and peripheral resistance (total peripheral resistance (TPR)) in hypertensives. In 80 subjects (age $52 \pm 13$ years; 53\% males; including 23 normotensives and 57 hypertensives, among which 29 were uncontrolled hypertensives), we used: (1) the new non-invasive RTX1 adaptive optics (AO) camera (Imagine Eyes, Orsay, France) to measure the wall-to-lumen ratio (WLR) on retinal microvasculature; (2) cardiovascular magnetic resonance (CMR) imaging to assess aortic stiffness, geometry and cardiac output; and (3) the validated SphymoCor Xcel device to measure central blood pressure (BP) and carotido-femoral pulse wave velocity (Cf-PWV). TPR was calculated as the central mean BP/cardiac output ratio. WLR and TPR were significantly higher and aortic distensibility was significantly lower in hypertensives. Aortic dilation and arch elongation were found in uncontrolled hypertensives. In the univariate analysis, WLR was positively correlated with central $B P(P<0.001)$, TPR $(P<0.001)$ and Cf-PWV $(P<0.05)$, and it was negatively correlated with aortic distensibility $(P=0.003)$; however, it was not correlated with age or cardiovascular risk factors. The multivariate analysis indicated that WLR was associated with TPR
\end{abstract} $(P=0.002)$ independent of age, BMI, gender, antihypertensive treatments, aortic diameter and central SBP. As expected, age was the major correlate of ascending aorta distensibility and Cf-PWV. New non-invasive vascular imaging methods are complementary for the detection of the deleterious effects of aging or high BP on large and small arteries. AO examination could represent a useful tool for the study and follow-up of microvasculature anatomical changes.

Hypertension Research (2016) 39, 536-542; doi:10.1038/hr.2016.26; published online 24 March 2016

Keywords: cardiovascular magnetic resonance; hypertension; microcirculation; optical imaging; remodeling

\section{INTRODUCTION}

Hypertension affects the structure and the function of both large and small arteries. The major hemodynamic determinants of blood pressure (BP) are cardiac output and total peripheral resistance (TPR), which reflect the vascular tone of small arteries. The vast majority of prescribed antihypertensive treatments (such as reninangiotensin system inhibitors or calcium channel blockers) decrease TPR via their vasorelaxant properties, and some studies have shown the value of using thoracic impedance-based methods that indirectly evaluate TPR to adapt antihypertensive treatments in uncontrolled hypertensive patients. ${ }^{1}$

In hypertensive subjects, an increase of the media-to-lumen-ratio (MLR) of the small arteries has been observed in s.c. arteries dissected from gluteal biopsies. ${ }^{2}$ More recently, a non-invasive approach for small artery imaging in vivo has been designed and validated. Using scanning laser Doppler flowmetry, ${ }^{3,4}$ the wall-to-lumen ratio (WLR) of retinal arterioles has been assessed, and associations between WLR and BP have been confirmed. More recently, adaptive optics (AO), which is a novel, accurate and reproducible opto-electronic method, has been used to non-invasively determine the qualitative and quantitative microvascular morphometry of small vessels at a near-histological scale in the human retina. ${ }^{5,6}$

Parallel to microvascular changes, large artery alterations in hypertension have been studied in depth using a wide panel of methods, including invasive catheterization, ultrasound, ${ }^{7}$ applanation tonometry ${ }^{8}$ and, more recently, cardiovascular magnetic resonance

${ }^{1}$ Preventive Cardiovascular Unit, Institute of Cardiometabolism and Nutrition, Groupe Hospitalier Universitaire Pitié-Salpêtrière, Assistance Publique-Hôpitaux de Paris, Paris, France; ${ }^{2}$ Sorbonne Universités, UPMC Univ Paris 06, INSERM 1146, - CNRS 7371, Laboratoire d'imagerie Biomédicale, Paris, France; ${ }^{3}$ Imaging Core Lab, Institute of Cardiometabolism and Nutrition, ICAN, Paris, France; ${ }^{4}$ Unité INSERM 968 Institut de la vision—Centre d'Investigation Clinique 503 Centre Hospitalier National des Quinze-Vingts, Assistance Publique-Hôpitaux de Paris, Paris, France and ${ }^{5}$ Département d'imagerie cardiovasculaire et de radiologie interventionnelle, Pôle Imagerie-Groupe Hospitalier Pitié-Salpêtrière, Assistance Publique-Hôpitaux de Paris, Paris, France

Correspondence: Dr D Rosenbaum, Unité INSERM 1146 - Laboratoire d'imagerie Biomédicale, Faculté de médecine Pierre et Marie Curie, 91 Boulevard de l'Hôpital, Paris 75013, France.

E-mail: david.rosenbaum@psl.aphp.fr

Received 9 November 2015; revised 20 January 2016; accepted 1 February 2016; published online 24 March 2016 
(CMR) imaging. Thanks to such modalities, stiffening, hemodynamics and geometrical changes of large arteries including the carotid arteries 9 and proximal aorta ${ }^{10}$ have been demonstrated. Recent studies indicated that a combination of central pressure measurements with CMR enables a comprehensive evaluation of the proximal aorta through reliable indices of geometry, ${ }^{10}$ distensibility, ${ }^{11,12}$ pulse wave velocity and TPR. ${ }^{13}$ Strong associations of these CMR indices with aging and cardiovascular events have been demonstrated. ${ }^{14}$

Our aim was to investigate the relationships between WLR, large artery characteristics and total peripheral resistance in the setting of arterial hypertension. To achieve this aim, we evaluated the characteristics of large and small arteries in normotensive and hypertensive patients using non-invasive imaging modalities such as CMR for large arteries and $\mathrm{AO}$ for small arteries.

\section{METHODS}

Between January 2013 and February 2014, hypertensive patients and controls were prospectively recruited in a dedicated outpatient clinic (cardiovascular prevention unit). This clinical study was carried out according to the principles outlined in the Declaration of Helsinki. Approval of the local ethics committee was obtained, and informed consent was provided by all participants.

The inclusion criteria for normotensives were no personal history of cardiovascular disease, no antihypertensive treatments and normal home blood pressure (systolic blood pressure (SBP) $<135 \mathrm{~mm} \mathrm{Hg}$ and diastolic blood pressure (DBP) $<85 \mathrm{~mm} \mathrm{Hg}$ ). The inclusion criteria for hypertensives were: the absence of secondary causes of hypertension; the absence of personal history of cardiovascular disease; and a stable regimen of antihypertensive treatment for at least 6 months with a therapy containing one or two of the following classes: $\mathrm{ARBs}$ or calcium blockers. If a third antihypertensive medication was present, it had to be a thiazide.

The exclusion criteria were diabetes, antihypertensive treatment with beta blockers, more than four cumulated antihypertensive treatments, contraindication to CMR exam and refusal to give consent.

Controlled hypertension was defined as having normal home blood pressure (SBP $<135 \mathrm{~mm} \mathrm{Hg}$ and DBP $<85 \mathrm{~mm} \mathrm{Hg}$ ).

\section{Study protocol}

During the outpatient visit: (1) basic characteristics were recorded, including age, weight, height and familial and personal history of cardiovascular disease; (2) venous blood samples were taken in the supine position to measure urea, creatinine and potassium; (3) peripheral and central BP measurements were taken; and (4) $\mathrm{AO}$ and $\mathrm{CMR}$ imaging were performed.

\section{Blood pressure assessment}

For each subject, two different measurements of BP were performed:

(1) Central BP measurements were performed in a temperature-controlled semi-dark room to reproduce the CMR exam conditions. Measurements were performed in standard conditions after $5 \mathrm{~min}$ of rest and $20 \mathrm{~min}$ before the CMR exam using the validated SphymoCor Xcel device (AtCor). ${ }^{15}$
(2) Subjects were asked to record their home BPs according to a standardized protocol using the validated OMRON M3 brachial cuff device ${ }^{16}$ during the 3 days preceding the outpatient visit.

Overall, the following BP measurements were recorded and considered for analysis:

- Home SBP, home DBP, home pulse pressure (PP) and home mean BP (MBP).

- Central systolic BP (cSBP), central diastolic BP (cDBP), central pulse pressure (cPP) and central mean BP (cMBP).

\section{Small arteries: WLR estimation by AO}

Retinal imaging was performed by an operator blinded to the clinical and CMR data at the Clinical Investigation Center of the Quinze-Vingts Hospital (Paris, France) within 2 weeks before or after CMR and central pressure measurements. En face $\mathrm{AO}$ fundus images were obtained using a commercially available flood-illumination AO retinal camera (rtxl; Imagine Eyes, Orsay, France).

Briefly, the rtx 1 camera measures and corrects wave front aberrations with a $750 \mathrm{~nm}$ super luminescent diode source and an $\mathrm{AO}$ system operating in a closed loop. A $4^{\circ} \times 4^{\circ}$ fundus area (i.e., approximately $1.2 \mathrm{~mm} \times 1.2 \mathrm{~mm}$ in emmetropic eyes) is illuminated at $840 \mathrm{~nm}$ by a temporally low-coherent lightemitting diode flashed flood source, and a stack of 40 fundus images is acquired in $4 \mathrm{~s}$ by a charge coupled device camera.

Among the 80 patients, 74 examinations were performed without pupil dilation. In six patients, pupil dilation was necessary and was obtained with topical tropicamide monodose $\left(2 \mathrm{mg} 0.4 \mathrm{ml}^{-1}\right.$, Novartis, France). These six examinations were excluded from the analysis that integrated retinal $\mathrm{AO}$ parameters. After $10 \mathrm{~min}$ of rest, the patient was situated on the chin rest. The live video image of the pupil allowed for alignment with the incident light. The live display of the AO-corrected fundus image allowed for adjustment of brightness, contrast and focus.

Gaze was oriented using a dedicated target to capture the region of interest, which included a segment of the superotemporal artery of the right eye, devoid of bifurcations, at least $250 \mu \mathrm{m}$ long and with an inner diameter of at least $50 \mu \mathrm{m}$ (see example in Figure 1). The site of interest was chosen to be free of the presence of focal arterial nicking (FAN) and arterio-venous crossings.

To identify the systolic pulse and delete the corresponding images, real-time videos were generated from each stack using customized imaging software. Then, the diastolic images were averaged to increase the signal-to-noise ratio. Such averaged diastolic AO images were semi-automatically processed using reproducible ${ }^{6}$ and previously described custom software. ${ }^{17}$ Image processing included image enhancement by applying a median filter followed by a nonlinear diffusion filter ${ }^{18}$ to smooth the blood vessels, while preserving the contrast along their edges, and a previously described image segmentation described in detail in Lermé et al. ${ }^{17,19}$ and based on mathematical morphology, k-means clustering and active contours models relying on parallelism information ${ }^{20}$ to extract the internal diameter (ID) and outer diameter (OD) of the vessel.

Finally, the ratio of total parietal thickness over the lumen diameter averaged along a $250 \mu \mathrm{m}$ length defined the WLR. Wall thickness (WT) was defined as $\mathrm{WT}=(\mathrm{OD}-\mathrm{ID}) / 2$, and total wall cross-sectional area (WCSA) was defined as
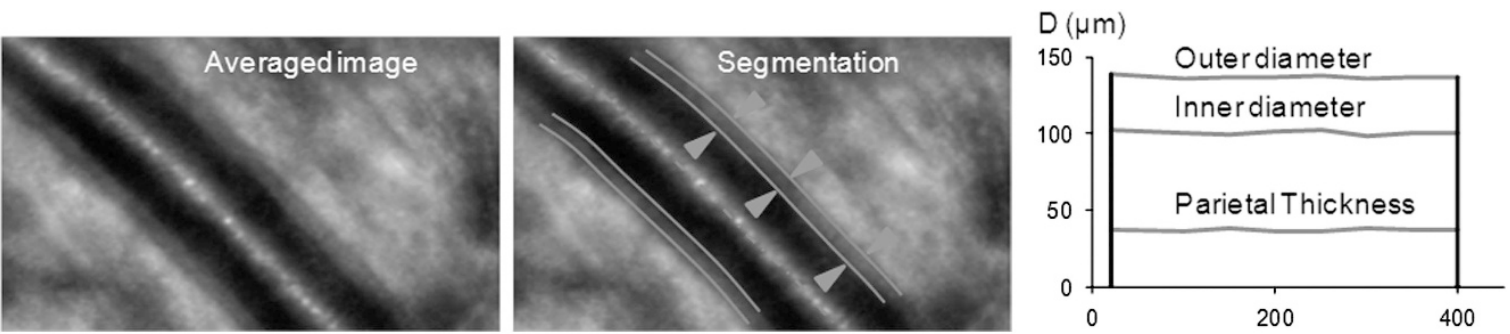

Figure $1 \mathrm{AO}$ imaging and segmentation of a retinal arteriole. Left: averaged image after acquisition. Middle: image segmentation. Note that parietal structures (between arrowheads) can be seen. $C$, morphogram of the segmented vessel ( $D$ : diameter; $L$ : length). 
WCSA $=\pi^{*}\left((\mathrm{OD} / 2)^{2}-(\mathrm{ID} / 2)^{2}\right)$. More details on the precision of the AO device and axial correction are presented in the Supplementary File.

\section{Characterization of large arteries}

Carotid-femoral pulse wave velocity. Carotid-femoral pulse wave velocity (Cf-PWV) was measured between the carotid and femoral locations using the validated SphygmoCor Xcel device and using the distance between the two locations and the transit time between the corresponding pressure waveforms. Cf-PWV was calculated as $\mathrm{PWV}=0.8(D / t){ }^{21}$ All calculations, including the measurement of parameters $>12$ cardiac cycles, were automated. The average of two high-quality recordings was used for Cf-PWV.

Proximal aortic stiffness and geometry indices by CMR. All patients underwent CMR on a clinical 1.5 T Aera Siemens magnet (Siemens Healthcare, Erlangen, Germany) with a 16-channel cardiac-phased array surface coil and ECG gating. Cine SSFP acquisitions and through-plane velocity encoded data were acquired in the ascending aorta (AA) at the level of the pulmonary bifurcation perpendicular to the aortic wall. Acquisition parameters for both aortic SSFP and velocity encoding data are provided in the Supplementary File.

Images were analyzed offline by an operator blinded to the clinical data. As previously described, ${ }^{22}$ contours of the AA were automatically detected on SSFP images for all phases of the cardiac cycle using the validated ArtFun software (UPMC-Inserm), ${ }^{12,22,23}$ providing AA area and diameter variations during the cardiac cycle. This method was also used for the automated detection of both $\mathrm{AA}$ and descending aorta contours from modulus images of PC sequences, which were superimposed on velocity images for AA flow analysis.
The automated aortic segmentation of SSFP and PC images, combined with the Sphygmocor Xcel central BP measurements, enabled the estimation of the following parameters:

(1) AA distensibility $(\mathrm{AAD})=($ As $-\mathrm{Ad}) /(\mathrm{Ad} \mathrm{cPP})$ as previously described, ${ }^{11}$ where As and Ad correspond to SSFP systolic and diastolic AA areas $\left(\mathrm{mm}^{2}\right)$ and $\mathrm{CPP}$ is the central pulse pressure. AA diastolic diameter was calculated from Ad.

(2) Aortic arch width defined as the distance between the center of the ascending and descending aorta cross-sections. ${ }^{10}$

(3) Aortic arch height defined as the length of the orthogonal projection of the inflection point of the aortic arch centerline, positioned at the top of the arch, on the width of the aortic arch. ${ }^{10}$

(4) Aortic arch length was estimated from SSFP acquisitions as the distance between the ascending and proximal descending aorta locations, as previously described. ${ }^{10}$

(5) Aortic arch pulse wave velocity (AAPWV) was estimated as the ratio between the previously mentioned arch length and the transit time between ascending and descending aorta flow waveforms as previously reported. ${ }^{11}$ Transit time was calculated automatically using a method based on the least-squares minimization approach between the systolic up-slope of the ascending and descending aorta flow curves (ArtFun software, UPMC, Laboratoire d'Imagerie Biomédicale, Paris, France).

(6) Cardiac output was calculated using heart rate during the CMR velocity acquisition multiplied by the stroke volume measured as the area under the ascending aorta flow curve.

Table 1 Description of subjects including basic characteristics, blood pressure measurements and anatomical and functional micro- and macro-vascular indices

\begin{tabular}{|c|c|c|c|c|}
\hline & Normotensives & Controlled hypertensives & Uncontrolled hypertensives & Across groups $\mathrm{P}$-value \\
\hline Subjects number & 23 & 28 & 29 & \\
\hline Age, years (s.d.) & $45.1(12.2)$ & $52.9(12.1)^{*}$ & $53.1(12.2)^{*}$ & 0.04 \\
\hline Antihypertensive treatment number, $n$ & $0(0)$ & $1.64(0.18)^{*}$ & $1.17(0.17)^{*}$ & $<0.0001$ \\
\hline \multicolumn{5}{|l|}{ Central blood pressure (mmHg) } \\
\hline SBP & $112.9(2.8)$ & $123.1(2.4)^{*}$ & $135.3(2.3)^{*} \dagger$ & $<0.0001$ \\
\hline DBP & $77.2(2.1)$ & $84.3(1.8)^{*}$ & $92.6(1.7)^{*} \dagger$ & $<0.0001$ \\
\hline MBP & $89.3(2.1)$ & $97.0(1.8)^{*}$ & $107.1(1.7)^{*} \dagger$ & $<0.0001$ \\
\hline PP & $36.1(1.9)$ & $39.5(1.8)$ & $43.4(1.7)^{*}$ & 0.02 \\
\hline \multicolumn{5}{|l|}{ Hemodynamics } \\
\hline Total peripheral resistance (dynes $\mathrm{s}^{-1} \mathrm{~cm}^{-5}$ ) & $1567(124)$ & $1801(108)$ & $2047(102)^{*}$ & 0.01 \\
\hline Cardiac output $\left(\mathrm{ml} \mathrm{s}^{-1}\right)$ & $78.7(9.7)$ & $76.3(8.8)$ & $84.4(8.8)$ & 0.8 \\
\hline \multicolumn{5}{|l|}{ Microvasculature } \\
\hline Retinal WLR & $0.276(0.012)$ & $0.304(0.011)$ & $0.343(0.010)^{*} \dagger$ & 0.0002 \\
\hline Retinal arteriolar internal diameter $(\mu \mathrm{m})$ & $83.7(14.4$ & $78.8(9.8)$ & $77.2(12.6)$ & 0.19 \\
\hline Retinal arteriolar wall thickness $(\mu \mathrm{m})$ & $22.0(2.32)$ & $22.1(3.9)$ & $25.1(4.3)^{*} \dagger$ & 0.04 \\
\hline Retinal arteriolar wall cross-sectional area $\left(\mu \mathrm{m}^{2}\right)$ & $3447(292.8)$ & $3278(213.8)$ & $3477(176.8)$ & 0.7 \\
\hline \multicolumn{5}{|l|}{ Aorta structure $(\mathrm{cm})$} \\
\hline Ascending aorta diastolic diameter & $2,57(0.06)$ & $2,93(0.07)^{*}$ & $3.09(0.06)^{*}$ & $<0.0001$ \\
\hline Aortic arch length & $118.4(4.9$ & $131.0(4.5)$ & $136.3(4.5)^{*}$ & $<0.0001$ \\
\hline Aortic arch width & $64.1(1.9)$ & $69.3(1.7)$ & $77.0(1.7) * \dagger$ & $<0.0001$ \\
\hline Aortic arch height & $39.7(2.0)$ & $41.8(1.8)$ & $44.6(1.8)^{*}$ & $<0.0001$ \\
\hline \multicolumn{5}{|l|}{ Aorta function } \\
\hline Ascending aorta distensibility $\left(\mathrm{kPa}^{-1} \times 10^{-3}\right)$ & $43.4(3.7)$ & $24.3(3.3)^{*}$ & $18.0(3.2)^{*}$ & $<0.0001$ \\
\hline Ascending aorta PWV $\left(\mathrm{m} \mathrm{s}^{-1}\right)$ & $5.37(0.61)$ & $7.25(0.54)^{*}$ & $8.55(0.53)^{*}$ & 0.001 \\
\hline Carotid-femoral PWV $\left(\mathrm{s}^{-1}\right)$ & $7.89(0.31)$ & $8.87(0.27)^{*}$ & $9.33(0.25)^{*}$ & 0.002 \\
\hline
\end{tabular}

Abbreviations: BMI, body mass index; PWV, pulse wave velocity; SBP, DBP and PP, systolic diastolic and pulse blood pressures; WLR, wall-to-lumen ratio.

${ }^{*} P$-value $<0.05$ at least vs. normotensives.

${ }^{\dagger} P$-value $<0.05$ at least $v s$. controlled hypertensive. 
Finally, total peripheral resistance (TPR) was calculated as the ratio between central mean BP and cardiac output. ${ }^{24}$

\section{Statistical analysis}

All continuous variables are expressed as the mean (s.d.), unless otherwise stated.

Differences between groups were evaluated by ANOVA and the nonparametric Kruskal-Wallis test. Distribution of categorical variables between groups was evaluated using the $\chi^{2}$ test. Correlations between two variables were assessed using a linear regression model, and Pearson's correlation coefficient $(r)$ was provided. The normality of the distribution of continuous variables was tested using Shapiro-Wilk's test. WLR and stiffness parameters (Cf-PWV and AAD) were further studied for their associations with age, body mass index, central SBP, TPR and antihypertensive treatments (any treatment $=1$, no treatment $=0$ ) using multivariate regression models, and partial correlations for independent determinants of WLR, AAD and Cf-PWV are provided. Prior to the multivariate analyses, variance inflation due to covariates was verified by estimating a variance inflation factor $<2$.

All statistical tests were two-tailed and a $P$-value $<0.05$ indicated statistical significance. All analyses were performed using SAS software, JMP10 (SAS Institute Inc., Cary, NC, USA).

\section{RESULTS}

Characteristics of normotensive subjects, controlled hypertensive patients and uncontrolled hypertensive patients enrolled in the study are shown in detail in Table 1 and in the Supplementary File. While normotensive subjects were younger than hypertensive patients, BMI, sex ratio, LDL-C, HbAlc, smoking status and creatinine were similar among the three groups (additional Table 1).

Table 2 Correlation coefficients resulting from univariate associations between retinal and wall-to-lumen ratio (WLR) as well as wall cross-sectional area (WCSA) and age, BMI, central blood pressures, large artery indices and total peripheral resistance

WLR

Demographic characteristics

Age (years)

0.14

$\mathrm{BMI}\left(\mathrm{kg} \mathrm{m}^{-2}\right)$

0.22

Blood pressure $(\mathrm{mmHg})$

Central SBP

Central DBP

Central. MBP

Central PP

$0.48^{\dagger}$

$0.50^{\ddagger}$

$0.51^{\ddagger}$

0.22

Aorta structure $(\mathrm{cm})$

Aortic arch length

0.17

Aortic arch width

Aortic arch height

0.06

Ascending aorta diastolic diameter

$0.39+$

\section{Aorta function}

Ascending aorta distensibility $\left(\mathrm{kPa}^{-1} \times 10^{-3}\right)$

Ascending aorta PWV $\left(\mathrm{m} \mathrm{s}^{-1}\right)$

Carotid-femoral PWV $\left(\mathrm{m} \mathrm{s}^{-1}\right)$

Peripheral resistance

Total peripheral resistance (dynes $\mathrm{s}^{-1} \mathrm{~cm}^{-5}$

Abbreviations: SBP, DBP, MBP and PP, systolic, diastolic, mean and pulse blood pressures;

WLR, wall-to-lumen ratio.

${ }^{*} P$-value $<0.05$ at least.

$P$-value $<0.01$ at least.

${ }^{\ddagger} P$-value $<0.001$ at least.
While central PP differed significantly only in uncontrolled hypertensive patients, a significant increase in all peripheral and central BP measurements was observed throughout the three groups, with the highest BP observed in uncontrolled hypertensive patients compared with controlled hypertensives or normotensive subjects.

The characteristics of the large and small arteries in the three groups are also summarized in Table 1. Regarding small arteries, retinal WLR as well as retinal arteriolar wall thickness were significantly higher in uncontrolled hypertensive patients than in controlled hypertensives and normotensive subjects, whereas retinal wall cross-sectional area (WCSA) did not significantly vary among the three groups. Moreover, a non-significant trend toward a diminution in the internal diameter was noted.

Regarding large arteries, aortic geometry indices, such as diameter and arch length, width and height, indicated a dilatation of the ascending aorta cross-section as well as a lengthening and an enlargement of the aortic arch in uncontrolled hypertensive patients. Finally, indices of arterial stiffness such as CMR ascending aorta distensibility and AAPWV as well as Cf-PWV were substantially and significantly different between the three groups.

Univariate associations of WLR with age, BMI, central BPs, large artery indices and TPR are summarized in Table 2. WLR was significantly associated with SBP, DBP and MBP $(P<0.0001)$ as well as with all aortic diameters and stiffness indices and TPR $(r=0.56$, $P<0.0001)$. Of note, among retinal arteriolar indices, WLR resulted in the highest correlation coefficients for such associations.

The multivariate analysis (Table 3) indicated that WLR was associated with TPR $(P=0.002)$ independent of age, BMI, gender, antihypertensive treatments, AA diameter and central SBP. For these analyses, partial correlation coefficients $R^{2}$ for independent variables summarized in Figure 2 indicate that TPR was the strongest predictor of WLR. TPR explained $42 \%$ of the full multivariate model variance and AA diameter 23\%. For AA distensibility and Cf-PWV, age explained 64 and 59\% of the full multivariate model variance, respectively (see additional figure in the Supplementary File). Of note, although stiffness indices were associated with WLR in the univariate analysis, such associations were no longer significant after adjustment for age and SBP.

\section{DISCUSSION}

In this study, we were able to show that the WLR of small arteries of the retina was correlated with $\mathrm{BP}$ regimen and total peripheral resistance in hypertension. We also showed that, if age and BP were the strongest correlates of morphological and functional changes in large arteries, total peripheral resistance was the strongest correlate of WLR, beyond central SBP and age. Finally, among large artery parameters, we showed that local CMR evaluation of proximal aortic geometry and stiffness is relevant in hypertensive subjects. Our results are in line with previous results showing the interdependence of hypertension and arterial stiffness. ${ }^{21}$ Here, in a population of patients with controlled and uncontrolled BP, we report a strong effect of age on stiffness but also on aortic anatomy. We extend the results of Redheuil et al. ${ }^{10,11}$ to hypertensive subjects, showing that $\mathrm{AAD}$ is an efficient integrator of age and BP consequences on stiffness. In our population, Cf-PWV was strongly associated with age but not with WLR or TPR. Accordingly, our findings on the aorta emphasize the prominent and confounding role of age on the properties of large arteries in hypertension.

Many studies in the literature report relationships between BP and microvasculature in humans. ${ }^{1}$ 
Table 3 Correlates of wall-to-lumen ratio

\begin{tabular}{|c|c|c|c|}
\hline & \multicolumn{3}{|c|}{ Wall-to-Iumen ratio } \\
\hline & $\mathrm{R}^{2}$ & $\beta$ (s.d.) & $P$ \\
\hline Overall model & 0.43 & & 0.0001 \\
\hline Age (years) & & $-0.0005(0.0006)$ & 0.36 \\
\hline Gender $($ male $=1)$ & & $-0.0112(0.0128)$ & 0.38 \\
\hline BMI (kg m $\left.{ }^{-2}\right)$ & & $0.0013(0.0013)$ & 0.34 \\
\hline cSBP (mmHg) & & $0.0005(0.0005)$ & 0.33 \\
\hline Antihypertensive treatment (for yes $=1$ ) & & $-0.0033(0.0158)$ & 0.83 \\
\hline $\begin{array}{l}\text { Total peripheral resistance } \\
\text { (dynes } \mathrm{s}^{-1} \mathrm{~cm}^{-5} \text { ) }\end{array}$ & & $5.4 .10-5(1.6 .10-5)$ & 0.002 \\
\hline AA diameter $(\mathrm{cm})$ & & $0.05(0.22)$ & 0.02 \\
\hline
\end{tabular}

Abbreviations: $\mathrm{AA}$, ascending aorta; $\mathrm{BMI}$, body mass index; $\mathrm{CSBP}$, central systolic blood pressure.

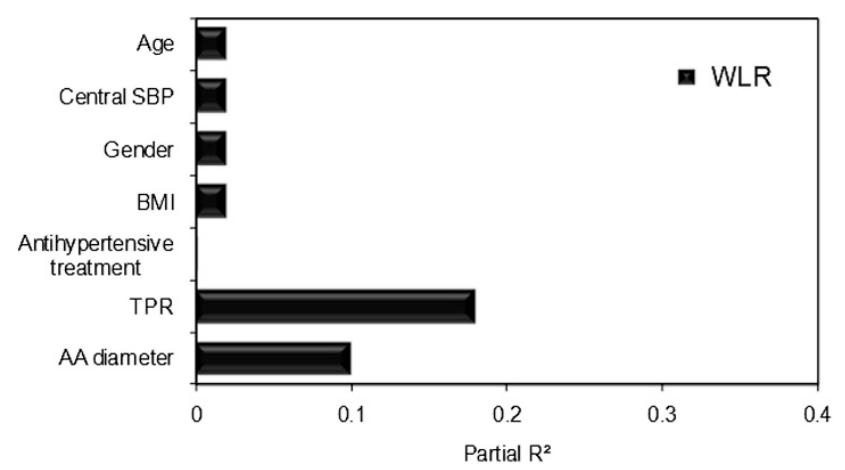

Figure 2 Determinants of WLR. Partial correlation coefficients $R^{2}$ are provided for each variable along with its statistical significance, which is indicated by ${ }^{*} P$-value $<0.05$, $† P$-value $<0.01$ or $\ddagger P$-value $<0.001$. AA, ascending aorta; BMI, body mass index; TPR, total peripheral resistance; cSBP, central systolic blood pressure.

In vitro studies by Schiffrin et al. ${ }^{2,25}$ have consistently shown strong relationships between elevated $\mathrm{BP}$ and increases in the media-tolumen-ratio (MLR) in s.c. arterioles. The non-invasive approach of microvasculature imaging in the retina using scanning laser Doppler flowmetry has provided further evidence of relationships between increased WLR and elevated BP and evidence of correlations between MLR and WLR has been shown. ${ }^{3}$ Recently, Salvetti et al. ${ }^{26}$ also reported relationships between $24 \mathrm{~h} \mathrm{BP}$, central BP and retinal WLR measured with scanning laser Doppler flowmetry. The strongest associations were found between daytime SBP, 24-h SBP and WLR. Here, we report that MBP, SBP and DBP are related to WLR, whereas $\mathrm{PP}$ is linked to AAD by definition. PP is an indicator of the pulsatile component of the BP periodic phenomenon. It is related to several hemodynamic mechanisms, of which notably compliance of large arteries is in compliance. In contrast, $\mathrm{MBP}$ is considered to represent the steady component of BP that is determined by cardiac output and vascular resistance. ${ }^{27}$ Another explanation for the lack of correlation between WLR and PP could be the relative youthfulness and the relative low PP in our population in comparison with other studies where associations with PP were reported. ${ }^{28}$

Little data have specifically reported the coupling between small and large arteries in hypertensives. In general populations, retinal arteriolar narrowing evaluated by the arteriole-to-venule ratio as measured in standard fundus photography has been linked to $\mathrm{AAD}$, independently of SBP, ${ }^{29}$ and aortic stiffness has been associated with the central retinal arteriolar and retinal vascular fractal dimension. ${ }^{30}$ Salvetti et $a l^{26}$ found that PWV was an independent determinant of WLR in a heterogeneous population of hypertensive/diabetic patients (treated and never treated). Here, in a homogeneous population of hypertensives where small and large arteries were characterized at the same moment, we demonstrated a correlation between the morphological indices of small and large arteries (WLR and ascending aorta diameter) that was independent of age, BP and antihypertensive treatment. This observation of a morphological continuum in different arterial beds extends the concept of interrelated changes in macro- and microvasculature. Additionally, while stiffness indices were associated with retinal eutrophic remodeling in the univariate analysis, such associations were no longer significant after adjustment for age and SBP. Nevertheless, TPR was shown to be the only independent determinant of WLR, explaining $42 \%$ of the variance of the multivariate model relating WLR with age, gender, BMI, antihypertensive treatments, central SBP, AA diameter and TPR.

Conversely to all large artery parameters and consistent with previous findings ${ }^{26,29-31}$ but not with our previous results, WLR was not related to age in this population. The first explanation would be the lack of power and the small effective size of our study. A second explanation could be that WLR is not a marker of aging but of remodeling, a dynamic phenomenon driven by pressure regimen. In essential hypertension, histological investigations of s.c. arteries have shown eutrophic remodeling characterized by reduced lumen diameter and increased MLR with no net increase in muscle mass. ${ }^{32}$ Here, we were able to reproduce those results using an entirely non-invasive method. Arteriolar lumen reduction can result from rearrangement of smooth muscle cells layers but it can also be secondary to vasoconstriction. Such hypotheses are in line with the study by Rizzoni et al. that reported an association between s.c. MLR and the vasodilatory capacity of coronary arteries, ${ }^{33}$ a result that can also be interpreted as being due to remodeling. ${ }^{34}$ In the retina, an association between capillary blood flow and the inner diameter of retinal arteries has been reported using scanning laser Doppler flowmetry. In this latter study, an inverse correlation between retinal arterial vasodilatory capacity and WLR in hypertensive patients was observed. ${ }^{35}$ Furthermore, in our previous report on retinal small arteries in hypertensive patients, as a result of the high-resolution of $\mathrm{AO}$, we showed that the inner and outer limits of the arteriolar wall maintained their parallelism at sites of FAN, suggesting that FANs were caused by focal vasoconstriction rather than by parietal growth. ${ }^{6}$ Moreover, a turnover of retinal $\mathrm{FAN}^{36}$ and rapid modifications of WLR after one month of lercanidipine treatment ${ }^{37}$ has been demonstrated. Such findings may illustrate a potential interplay between structural remodeling and vascular tone in small arteries.

Although several non-invasive methods have been proposed to assess cardiac output, the direct thermodilution method remains the gold standard to assess cardiac output although it requires invasive catheterization. $^{38}$ Recently, an impedance-based device has been validated on intensive care unit patients, ${ }^{39}$ but the accuracy of impedance cardiography has been challenged. ${ }^{40}$ Here, we used an entirely non-invasive method ${ }^{13}$ to calculate resistance by combining central pressure measurements with the Sphygmocor Xcel device and direct measurement of aortic flow by CMR. WLR is a static parameter whose components are lumen diameter and wall thickness. From our experience with a large population, lumen diameter varies after a few days and wall thickness varies after a few weeks in the case of treatment inducing a drop in BP. Our results suggest that arterioles 
$<80 \mu \mathrm{m}$ assessed with the AO camera are resistive vascular territories that potentially determine TPR. However, when considering the physiological link between TPR and vascular anatomy, lumen diameter should be the main determinant of TPR. Here, TPR was found to be correlated with WLR but not with lumen diameter. This result may be explained by the fact that: (1) the diameter of arterioles assessed with AO would not be directly responsible for TPR and (2) it has been admitted from previous studies on s.c. biopsies and confirmed by our personal data that WLR is constant along the vascular tree in a microcirculatory territory.

Increased WLR in the retina, carotid IMT and aortic stiffness predict target organ damage and cardiovascular events in patients with hypertension; ${ }^{41}$ however, so far, none has been evaluated as a therapeutic tool in antihypertensive treatment management. However, impedance measurements have already been successfully used for personalized management of resistant hypertension. ${ }^{42}$ Furthermore, while a total normalization of alterations in small arteries was found in hypertensive patients treated with angiotensin-converting enzyme inhibitors or calcium blockers, normalization was incomplete or absent in patients treated with beta blockers or diuretics. ${ }^{43}$ In this context, AO WLR, due to its reproducibility and its non-invasive nature as well as its strong association with resistance, could be of great use in the follow-up of arteriolar tree tonus and morphological modifications under antihypertensive treatments.

Considering cardiovascular risk assessment, current markers and risk equations are poor predictors of cerebrovascular events, whereas Ota et al. showed that hypertensive retinopathy features predicted cerebrovascular events. ${ }^{44}$ Evidence is also increasing regarding structural retinal microvasculature changes being associated with cardiovascular risk even early in life ${ }^{45}$ as well as with emerging subclinical MRI brain infarcts and white matter lesions. ${ }^{46}$ In line with such findings, WLR could represent a potential predictor of cardiovascular events in hypertensives.

Our study has several limitations. First of all, because of the cross-sectional design of our study, we can only describe associations, and no conclusions of causality can be drawn.

Second, we included a relatively small number of patients. This is certainly the main explanation for the lack of an association between WLR and age. However, this enabled the construction of homogeneous and comparable groups that were well characterized, while avoiding potential biases such as diabetes, smoking status or gender imbalance. Only age was slightly different between the groups but this was accounted for in the multivariate analysis by age adjustment. The third limitation is on BP level assessment and timing. Central BP was recorded at the CMR time and not during the $\mathrm{AO}$ examination but both were very close in time and performed under the same conditions. Neither night-time BP nor $24 \mathrm{~h}$ central BP were recorded; however, home daytime-BP, which was used in our study, has previously been shown to be the strongest predictor of WLR. ${ }^{26}$ Another limitation is the exclusion of regions with FAN in the AO images analysis. We previously demonstrated that WLR in FAN regions was significantly increased. ${ }^{6}$ Still, despite the analysis in FAN-free areas, we were able to show a significant increase in WLR in uncontrolled hypertensive subjects.

Although it is well known that endothelial dysfunction is an initial step of the process of athero- and arteriosclerosis in hypertensives, the evaluation of endothelial function was unfortunately not performed in our study. Blood samples for Hs-CRP were taken and, while Hs-CRP was slightly higher in the hypertensive group, no independent associations with WLR were found in our study group (data not shown).
Additionally, we studied patients under antihypertensive treatment. All patients were treated with a stable regimen of similar vasodilators. This may have blurred some correlations, especially correlations with resistance, and it may have hampered the identification of medication effects on WLR. Moreover, due to the small size of our sample population, we were not able to address potential effects of specific antihypertensive drug classes. Retinal microvasculature WLR is known to be elevated in diabetes because of hypertrophic remodeling. Accordingly, it would have been interesting to compare such a population with hypertensives. However, type 2 diabetic patients often display other risk factors, which may have hampered comparisons in a small population.

Our results highlight the differential meaning between macro- and microvascular indices. While cf-PWV and AAD are markers of arterial aging in proximal large arteries, WLR of the retinal arterioles is a marker of BP regimen, total resistance and remodeling of the distal arterial tree. Consequently, these different markers may be useful to address different therapeutic targets. Quantitative, qualitative, in vivo and non-invasive characterization by $\mathrm{AO}$ and $\mathrm{CMR}$ imaging contribute to a better understanding of the consequences of hypertension on large and small arteries. AO examination is a quick, reproducible and non-invasive technique that could represent a useful tool to follow short- and long-term changes in microvasculature associated with antihypertensive treatments. Beyond WLR measurements, AO will allow for the examination of several arteriolar characteristics such as FAN, arterio-venous nicking, wall thickness regularity and bifurcation conformation. Future studies should be performed to determine the relative usefulness of micro- and macro-arterial markers with respect to patient outcome and management strategies.

\section{CONFLICT OF INTEREST}

The authors declare no conflict of interest.

\section{ACKNOWLEDGEMENTS}

ImagineEye provided technical assistance with image processing. Supported by the Institut National de la Sante' et de la Recherche Médicale (Contrat d'Interface 2011), the Agence Nationale de la Recherche (ANR-09-TECS-009 and ANR-12-TECS-0015-03), the Association Contre l'OVR (www.asso-ovr.fr), the Cardiometabolism and Nutrition Institute (http://www.ican-institute.org/) and the Société Française d'Hypertension (www.sfhta.fr). The funding organizations had no role in the design or conduct of this research. All authors take responsibility for all aspects of the reliability and freedom from bias of the data presented and their discussed interpretation.

Author contributions: DR: study conception, study realization, CMR image analysis, statistics and manuscript writing. NK: image analysis, study realization, statistics and manuscript writing. XG: study conception, study supervision, and manuscript supervision. AR: study conception, CMR acquisition and analysis and manuscript supervision. MP: study conception, OA image acquisition and manuscript supervision. EK: OA image acquisition and analysis. PC: CMR acquisition, study supervisionstudy conception, study realization, CMR image analysis, statistics and manuscript writing.

1 Taler SJ. Individualizing antihypertensive combination therapies: clinical and hemodynamic considerations. Curr Hypertens Rep 2014; 16: 451

2 Schiffrin EL, Hayoz D. How to assess vascular remodelling in small and medium-sized muscular arteries in humans. J Hypertens 1997; 15: 571-584.

3 Rizzoni D, Porteri E, Duse S, De Ciuceis C, Rosei CA, La Boria E, Semeraro F, Costagliola C, Sebastiani A, Danzi P, Tiberio GAM, Giulini SM, Docchio F, Sansoni G, Sarkar A, Rosei EA. Relationship between media-to-lumen ratio of subcutaneous small arteries and wall-to-lumen ratio of retinal arterioles evaluated noninvasively by scanning laser Doppler flowmetry. J Hypertens 2012; 30: 1169-1175.

4 Lehmann MV, Schmieder RE. Remodeling of retinal small arteries in hypertension. Am J Hypertens 2011; 24: 1267-1273. 
5 Rosenbaum D, Koch E, Girerd X, Rossant F, Pâques M. [Imaging of retinal arteries with adaptative optics, feasibility and reproducibility]. Ann Cardiol Angéiologie 2013; 62: 184-188.

6 Koch E, Rosenbaum D, Brolly A, Sahel J-A, Chaumet-Riffaud P, Girerd X, Rossant F, Paques M. Morphometric analysis of small arteries in the human retina using adaptive optics imaging: relationship with blood pressure and focal vascular changes. J Hypertens 2014; 32: 890-898.

7 Segers P, Rabben SI, De Backer J, De Sutter J, Gillebert TC, Van Bortel L, Verdonck P. Functional analysis of the common carotid artery: relative distension differences over the vessel wall measured in vivo. J Hypertens 2004; 22: 973-981.

8 Laurent S. Arterial stiffness in arterial hypertension. Curr Hypertens Rep 2006; 8: 179-180.

9 Bots ML, Groenewegen KA, Anderson TJ, Britton AR, Dekker JM, Engström G, Evans GW, de Graaf J, Grobbee DE, Hedblad B, Hofman A, Holewijn S, Ikeda A, Kavousi M, Kitagawa K, Kitamura A, Ikram MA, Lonn EM, Lorenz MW, Mathiesen EB, Nijpels G, Okazaki S, O'Leary DH, Polak JF, Price JF, Robertson C, Rembold CM, Rosvall M, Rundek T, Salonen JT, Sitzer M, Stehouwer CDA, Franco OH, Peters SAE, den Ruijter HM. Common carotid intima-media thickness measurements do not improve cardiovascular risk prediction in individuals with elevated blood pressure: The USE-IMT Collaboration. Hypertension 2014; 63: 1173-1181.

10 Redheuil A, Yu W-C, Mousseaux E, Harouni AA, Kachenoura N, Wu CO, Bluemke D, Lima JAC. Age-related changes in aortic arch geometry: relationship with proximal aortic function and left ventricular mass and remodeling. J Am Coll Cardiol 2011; 58: 1262-1270.

11 Redheuil A, Yu W-C, Wu CO, Mousseaux E, de Cesare A, Yan R, Kachenoura N, Bluemke D, Lima JAC. Reduced ascending aortic strain and distensibility: earliest manifestations of vascular aging in humans. Hypertension 2010; 55: 319-326.

12 Dogui A, Kachenoura N, Frouin F, Lefort M, De Cesare A, Mousseaux E, Herment A. Consistency of aortic distensibility and pulse wave velocity estimates with respect to the Bramwell-Hill theoretical model: a cardiovascular magnetic resonance study. J Cardiovasc Magn Reson Off J Soc Cardiovasc Magn Reson 2011; 13: 11.

13 Bargiotas I, Bollache E, Mousseaux E, Giron A, de Cesare A, Redheuil A, Kachenoura N. MR and applanation tonometry derived aortic impedance: association with aging and left ventricular remodeling. J Magn Reson Imaging JMRI 2014; 41: 781-787

14 Redheuil A, Wu CO, Kachenoura N, Ohyama Y, Yan RT, Bertoni AG, Hundley GW, Duprez DA, Jacobs DR, Daniels LB, Darwin C, Sibley C, Bluemke DA, Lima JAC. Proximal aortic distensibility is an independent predictor of all-cause mortality and incident CV events: the MESA study. J Am Coll Cardiol 2014; 64: 2619-2629.

15 Hwang MH, Yoo JK, Kim HK, Hwang CL, Mackay K, Hemstreet O, Nichols WW, Christou DD. Validity and reliability of aortic pulse wave velocity and augmentation index determined by the new cuff-based SphygmoCor Xcel. J Hum Hypertens 2014; 28 : 475-481.

16 Topouchian J, Agnoletti D, Blacher J, Youssef A, Ibanez I, Khabouth J, Khawaja S, Beaino L, Asmar R. Validation of four automatic devices for self-measurement of blood pressure according to the international protocol of the European Society of Hypertension. Vasc Health Risk Manag 2011; 7: 709-717.

17 Lermé N, Rossant F, Bloch I, Paques M, Koch E. Segmentation of retinal arteries in adaptiveoptics images. In International Conference on Pattern Recognition (ICPR), Stockholm, Sweden, 2014, pp 574-578.

18 Weickert J, Romeny BH, Viergever MA. Efficient and reliable schemes for nonlinear diffusion filtering. IEEE Trans Image Process Publ IEEE Signal Process Soc 1998; 7: 398-410.

19 Lermé N, Rossant F, Bloch I, Paques M, Koch E, Benesty J. A fully automatic method for segmenting retinal artery walls in adaptive optics images. Pattern Recognit Lett (doi:10.1016/j.patrec.2015.10.011).

20 Rossant F, Bloch I, Ghorbel I, Paques M. Parallel Double Snakes. Application to the segmentation of retinal layers in 2D-OCT for pathological subjects. Pattern Recognit 2015; 48: 3857-3870.

21 Van Bortel LM, Laurent S, Boutouyrie P, Chowienczyk P, Cruickshank JK, De Backer T, Filipovsky J, Huybrechts S, Mattace-Raso FUS, Protogerou AD, Schillaci G, Segers P, Vermeersch S, Weber T. Artery Society, European Society of Hypertension Working Group on Vascular Structure and Function, European Network for Noninvasive Investigation of Large Arteries. Expert consensus document on the measurement of aortic stiffness in daily practice using carotid-femoral pulse wave velocity. J Hypertens 2012; 30: 445-448.

22 Herment A, Kachenoura N, Lefort M, Bensalah M, Dogui A, Frouin F, Mousseaux E, De Cesare A. Automated segmentation of the aorta from phase contrast MR images: validation against expert tracing in healthy volunteers and in patients with a dilated aorta. J Magn Reson Imaging JMRI 2010; 31: 881-888.

23 Bollache E, Kachenoura N, Redheuil A, Frouin F, Mousseaux E, Recho P, Lucor D. Descending aorta subject-specific one-dimensional model validated against in vivo data. J Biomech 2014; 47: 424-431.
24 Segers P, Rietzschel ER, De Buyzere ML, Vermeersch SJ, De Bacquer D, Van Bortel LM, De Backer G, Gillebert TC, Verdonck PR. Asklepios investigators. Noninvasive (input) impedance, pulse wave velocity, and wave reflection in healthy middle-aged men and women. Hypertension 2007; 49: 1248-1255.

25 Schiffrin EL. Vascular remodeling in hypertension: mechanisms and treatment. Hypertension 2012; 59: 367-374.

26 Salvetti M, Agabiti Rosei C, Paini A, Aggiusti C, Cancarini A, Duse S, Semeraro F, Rizzoni D, Agabiti Rosei E, Muiesan ML. Relationship of wall-to-lumen ratio of retinal arterioles with clinic and 24-hour blood pressure. Hypertension 2014; 63: 1110-1115.

27 Darne B, Girerd X, Safar M, Cambien F, Guize L. Pulsatile versus steady component of blood pressure: a cross-sectional analysis and a prospective analysis on cardiovascular mortality. Hypertension 1989; 13: 392-400.

28 Ott C, Raff U, Harazny JM, Michelson G, Schmieder RE. Central pulse pressure is an independent determinant of vascular remodeling in the retinal circulation. Hypertension 2013; 61: 1340-1345.

29 Cheung N, Sharrett AR, Klein R, Criqui MH, Islam FMA, Macura KJ, Cotch MF, Klein BEK, Wong TY. Aortic distensibility and retinal arteriolar narrowing: the multi-ethnic study of atherosclerosis. Hypertension 2007; 50: 617-622.

30 Lin F, Zhu P, Huang F, Li Q, Yuan Y, Gao Z, Yu P, Lin J, Chen F. Aortic stiffness is associated with the central retinal arteriolar equivalent and retinal vascular fractal dimension in a population along the southeastern coast of China. Hypertens Res Off J Jpn Soc Hypertens 2015; 38: 342-348.

31 Ritt M, Harazny JM, Ott C, Schlaich MP, Schneider MP, Michelson G, Schmieder RE. Analysis of retinal arteriolar structure in never-treated patients with essential hypertension. J Hypertens 2008; 26: 1427-1434.

32 Korsgaard N, Aalkjaer C, Heagerty AM, Izzard AS, Mulvany MJ. Histology of subcutaneous small arteries from patients with essential hypertension. Hypertension 1993; 22: 523-526.

33 Rizzoni D, Palombo C, Porteri E, Muiesan ML, Kozàkovà M, La Canna G, Nardi M, Guelfi D, Salvetti M, Morizzo C, Vittone F, Rosei EA. Relationships between coronary flow vasodilator capacity and small artery remodelling in hypertensive patients. J Hypertens 2003; 21: 625-631.

34 Folkow B. Physiological aspects of primary hypertension. Physiol Rev 1982; 62: 347-504.

35 Ritt M, Harazny JM, Ott C, Raff U, Lehmann M, Michelson G, Schmieder RE. Influence of blood flow on arteriolar wall-to-lumen ratio in the human retinal circulation in vivo. Microvasc Res 2012; 83: 111-117.

36 Liew G, Campbell S, Klein R, Klein BEK, Sharrett AR, Cotch MF, Wang JJ, Wong TY. Ten-year longitudinal changes in retinal microvascular lesions: the atherosclerosis risk in communities study. Ophthalmology 2011; 118: 1612-1618.

37 De Ciuceis C, Salvetti M, Rossini C, Muiesan ML, Paini A, Duse S, La Boria E, Semeraro F, Cancarini A, Rosei CA, Sarkar A, Ruggeri G, Caimi L, Ricotta D, Rizzoni D, Rosei EA. Effect of antihypertensive treatment on microvascular structure, central blood pressure and oxidative stress in patients with mild essential hypertension. J Hypertens 2014; 32: 565-574.

38 Alhashemi JA, Cecconi M, Hofer CK. Cardiac output monitoring: an integrative perspective. Crit Care Lond Eng/ 2011; 15: 214.

39 Faini A, Omboni S, Tifrea M, Bubenek S, Lazar O, Parati G. Cardiac index assessment: validation of a new non-invasive very low current thoracic bioimpedance device by thermodilution. Blood Press 2014; 23: 102-108.

40 Raaijmakers E, Faes TJ, Scholten RJ, Goovaerts HG, Heethaar RM. A meta-analysis of three decades of validating thoracic impedance cardiography. Crit Care Med 1999; 27: 1203-1213.

41 Laurent S, Boutouyrie P, Asmar R, Gautier I, Laloux B, Guize L, Ducimetiere P, Benetos A. Aortic stiffness is an independent predictor of all-cause and cardiovascular mortality in hypertensive patients. Hypertension 2001; 37: 1236-1241.

42 Fadl Elmula FEM, Hoffmann P, Larstorp AC, Fossum E, Brekke M, Kjeldsen SE, Giønnæss E, Hiørnholm U, Kjær VN, Rostrup M, Os I, Stenehjem A, Høieggen A. Adjusted drug treatment is superior to renal sympathetic denervation in patients with true treatment-resistant hypertension. Hypertension 2014; 63: 991-999.

43 Agabiti-Rosei E, Rizzoni D. Regression of small resistance artery structural alterations in hypertension by appropriate antihypertensive treatment. Curr Hypertens Rep 2010; 12: $80-85$.

44 Sairenchi T, Iso H, Yamagishi K, Irie F, Okubo Y, Gunji J, Muto T, Ota H. Mild retinopathy is a risk factor for cardiovascular mortality in Japanese with and without hypertension: the Ibaraki Prefectural Health Study. Circulation 2011; 124: 2502-2511.

$45 \mathrm{Li}$ L-J, Kamran MI, Wong TY. Retinal vascular imaging in early life: Insights into processes and risk of cardiovascular disease. J Physiol (e-pub ahead of print 23 November 2015; doi:10.1113/JP270947).

46 Cheung N, Mosley T, Islam A, Kawasaki R, Sharrett AR, Klein R, Coker LH, Knopman DS, Shibata DK, Catellier D, Wong TY. Retinal microvascular abnormalities and subclinical magnetic resonance imaging brain infarct: a prospective study. Brain $J$ Neurol 2010; 133: 1987-1993.

Supplementary Information accompanies the paper on Hypertension Research website (http://www.nature.com/hr) 